\title{
Effect of Some Fertilizer Treatments on the Growth of Fountain Grass (Pennisetum Setaceum) Grown Under the Stress of Different Levels of Salinity
}

\author{
Neima F. Elghazaly ${ }^{1}$ Khaled A. Emam ${ }^{1}$ and Warda A. Aly ${ }^{2}$ \\ ${ }^{1}$ Botanical Garden Res. Dept., Hort. Rese. Inst. Agric. Res. Center, Giza, Egypt. \\ ${ }^{2}$ Ornamental Plants Res. Dept., Hort. Rese. Inst. Agric. Res. Center, Giza, Egypt.
}

Received: 20 Oct. 2020 / Accepted 10 Dec. 2020 / Publication date: 20 Dec. 2020

\begin{abstract}
A field experiment was carried out throughout two seasons (2018/2019 and 2019/2020) at the Location on Cairo Ismailia Desert Road, Egypt, to find out the effect of some fertilizer treatments on the growth of fountain grass (Pennisetum setaceum), grown under the stress of different levels of salinity and three treatments (potassium silicate, carboxylic acid and control), growing medium consists of mixture of sand + compost $(3: 1, \mathrm{v} / \mathrm{v})$. The effect of treatments on the growth and chemical composition of fountain grass was investigated. The highest level of saline water $4000 \mathrm{ppm} \mathrm{NaCl}$ reduced all parameters compared with 2000ppm NaCl. Moreover, soil additives and foliar application significantly increased parameters of both saline concentrations, potassium silicate, carboxylic acid and interaction between them has increased all growth parameters, plant height $(\mathrm{cm}$.$) , no. of tillers /Plant, No. of inflorescences/Plant, inflorescences length (\mathrm{cm})$, Herb fresh weight/plant (g), Herb dry weight/plant, and chemical components, total carbohydrates and $\mathrm{N} \%, \mathrm{P} \%$ and $\mathrm{K} \%$. However, the obtained results indicated that applied (4g.) potassium silicate as soil drench with carboxylic acid as foliar application had a positive effect on most of the growth parameters and chemical components.
\end{abstract}

Keywords: Pennisetum setaceum, fountain grass, stress, Salinity

\section{Introduction}

Pennisetum setaceum (fountain grass, Poaceae) is an apomictic, wind dispersed, C4 perennial bunch grass, native to Mediterranean parts of North Africa and the Middle East. It has successfully escaped cultivation and has invaded and naturalized in a wide range of habitats worldwide including Hawaii (Williams et al., 1995). Pennisetum setaceum is an invasive perennial grass found along roadsides and other disturbed sites in South Africa. Its successful spread is probably due to its popularity in horticulture, drought tolerance, unpalatability to animals, rapid growth and profuse seed production (Milton et al., 1998; Cabin et al., 2000). Red fountain grass is an ornamental grass that is used in the landscape for its reddish foliage and showy flowers and has been considered a valuable accent or specimen plant used around a fountain for the cooling silhouette it presents. The leaves of this 3- to 4-foot-tall plant are narrow, curving, linear blades with a beautiful red cast. The purple-pink or copper colored inflorescence of red fountain grass sits atop a hollow, 3-foot-long, nodding flower stalk. The feathery inflorescence is 12 inches long and occurs from early summer to frost. The fruits of this grass are small seeds that volunteer to form small plantlets nearby; this plant is considered mildly invasive. This plant is also lovely when planted in mass, and it makes a nice accent in a border. Flowers can be cut for fresh arrangements but fall apart as they dry, making them unsuited for dry arrangements. Plants grow poorly in the partial shade unless the soil has been cleared of roots surrounding trees and shrubs (Edward 1999).

Soil salinity is one of the major problems of agriculture in Egypt. Plants grown in the presence of excess salt suffer from oxidative stress, osmotic regulation, and ionic imbalance, which weaken plant metabolism and growth, (Amal, et al., 2007). Many approaches have been adopted to find the best horticultural practices to solve this problem. Recently, the use of salicylic acid and silicon had beneficial effects that increase the tolerance of plants to the unfavorable environment (Epstein and Bloom, 2003).

Potassium Silicate plays an active role in combating fungal growth by the production of polyphenolic compounds, this is a main part of the plants natural defense against fugal and insect 
attacks. Potassium silicate is a source of highly soluble potassium and silicon. It is used in agricultural production systems primarily as a silica amendment, and has the added benefit of supplying small amounts of potassium, increased tolerance of environmental stress, heat, cold, drought, water and soil toxicity or deficiency, improved growth rate both in the root zone and in the plant foliage. By using Potassium Silicate with clones we can increase the rate of roots kicked by as much as $80 \%$ (Marschner, 1995). In horticulture, potassium silicate is used as a soluble source of potassium and silicon. It makes the growing medium more alkaline. It is also used as a supplement (in conjunction with normal fertilizer) for the numerous benefits that increase the availability of silicon compounds. Silicon-containing compounds are valuable to a plant, and serve to support the plant. Stems thicken, the plant becomes more tolerant to drought and resists wilting, and the plant gets larger leaves and fruit (because the stem can support more weight (Wang and Galletta, 1998).

A carboxylic acid is an organic compound that contains a carboxyl group $(\mathrm{C}(=\mathrm{O}) \mathrm{OH})$, (Gold Book, 2014). The general formula of a carboxylic acid is $\mathrm{R}-\mathrm{COOH}$, with $\mathrm{R}$ referring to the rest of the (possibly quite large) molecule. Carboxylic acids occur widely and include the amino acids (which make up proteins) and acetic acid (which is part of vinegar and occurs in metabolism). Carboxylic acids as a type of organic acids contain carboxyl group which has a high chemical activity which influences the soil properties and soil solution by decreasing the $\mathrm{pH}$ number in soil which increases the availability of most minerals in soil with high $\mathrm{pH}$ number as calcareous and sandy soils (William and Jerry 2017). The relative proportion of aliphatic carboxylic acids varies for different soils (Dormaar, 1982).

The purpose of present research was to examine the Effect of some fertilizer treatments on Pennisetum setacum that was grown under two different levels of water salinity.

\section{Materials and Methods}

The experimental trial was performed throughout two successive seasons (2018/2019 and 2019/2020) at the Location on Cairo Ismailia Desert Road, Egypt to find out the effect of some fertilizers treatments on growth of fountain grass (Pennisetum setaceum) that was grown under stress of different levels of salinity. The second season was an extract repetition of the first one.

Materials:

- Seedlings of 25-30 cm. length were selected in March in both seasons.

- Growing medium of the mixture of sand + compost (3:1, v/v) was prepared for planting. The chemical properties of the growing medium are presented in Table.1 $(a, b)$. The analysis was carried out according to the methods described by Cottenie et al. (1982).

- Saline water ( $2 \mathrm{~g}$ from $\mathrm{NaCl}$ were dissolved in 1 liter water for preparing saline solution at the level of 2000ppm \& $4 \mathrm{~g}$ from $\mathrm{NaCl}$ were dissolved in 1 liter water for preparing saline solution at the level of $4000 \mathrm{ppm})$.

- Two types of fertilization:

- Potassium silicate has the formula $\mathrm{K}_{2} \mathrm{SiO}_{3}$, as a mineral fertilizer.

- Carboxylic acid as an organic fertilizer.

\section{Procedure:}

The seedlings were planted in $25-30 \mathrm{~cm}$ diameter plastic pots filled with the mixture of sand/ compost $(3: 1, \mathrm{v} / \mathrm{v})$ as one seedling per pot., in both seasons. Then plants were irrigated with two levels of saline water $(\mathrm{NaCl} 2000 \mathrm{ppm}$ and $4000 \mathrm{ppm} / \mathrm{l})$. After one month from planting the plants were treated with different two levels as a soil drench (potassium silicate 2, 4 g per plant) and (carboxylic acid 1, $2 \mathrm{~cm} / 1$ foliar application). Besides, untreated control plants, which were sprayed with tap water only. The different fertilization treatments were applied ten times during the life cycle of the plant under open field conditions at 15 days interval commencing from one month from planting. The layout of the experiment was randomized complete block design (RCBD) with three replicates. Every treatment contained 27 plants replicated three times $(9$ plants per experimental unit). needed.

Regular agricultural practices such as irrigation, weeding ... etc were carried out whenever 
The following data were recorded: Vegetative growth plant height $(\mathrm{cm}$.$) , No. of tillers /Plant, No.$ of inflorescences/Plant, inflorescences length $(\mathrm{cm})$, Herb fresh weight/plant $(\mathrm{g})$, Herb dry weight/plant.

\section{Chemical composition of the leaves:}

Total carbohydrates $(\mathrm{mg} / \mathrm{g}$ dry weight) were determined in the plant dry leaves of the different treatments according to Smith et al. (1956) and nitrogen (Pregel, 1945), phosphorus (Luatanab and Olseen 1965), potassium using flame photometer (Devis and Freitas, 1970).

Data were then tabulated and statistically analyzed using SAS program (1994) and means were compared by L.S.D. method according to Snedecor and Cochran (1980).

Table 1: (a). Chemical composition of extracted soil sample (water extract 1:2.5)

\begin{tabular}{|c|c|c|c|c|c|c|c|c|c|c|}
\hline \multirow[b]{2}{*}{ Soil media } & \multirow{2}{*}{$\begin{array}{l}\text { TDS } \\
\text { (Mg/l) }\end{array}$} & \multirow[b]{2}{*}{ pH } & \multirow{2}{*}{$\begin{array}{l}\text { E.C. } \\
\left(\text { Dsm }^{-1}\right)\end{array}$} & \multicolumn{4}{|c|}{ Cations (meq/l) } & \multicolumn{3}{|c|}{ Anions (meq/l) } \\
\hline & & & & $\mathrm{Ca}++$ & Mg++ & $\mathrm{Na}+$ & $\mathbf{K}+$ & HCO3- & $\mathrm{Cl}-$ & SO4- \\
\hline Sweet Sand & 370.1 & 8.3 & 0.658 & 2.3 & 0.2 & 3.2 & 0.026 & Nil & 4.4 & 1.4 \\
\hline
\end{tabular}

(b). Chemical properties of the used compost.

\begin{tabular}{cccccccccccccccc}
\hline $\begin{array}{c}\text { Organic } \\
\text { additive } \\
\text { type }\end{array}$ & \multicolumn{4}{c}{ Macro elements \% } & \multicolumn{6}{c}{ Micro elements (ppm) } & $\begin{array}{c}\text { O.C. } \\
\text { \% }\end{array}$ & $\begin{array}{c}\text { O.M } \\
\%\end{array}$ & $\begin{array}{c}\text { C/N } \\
\text { Ratio }\end{array}$ & pH & $\begin{array}{c}\text { E.C. } \\
\mathbf{d S m}^{1}\end{array}$ \\
\hline Compost & 1.41 & $\mathbf{P}$ & $\mathbf{K}$ & $\mathbf{C a}$ & $\mathbf{M g}$ & $\mathbf{Z n}$ & $\mathbf{F e}$ & $\mathbf{M n}$ & $\mathbf{C u}$ & \multirow{2}{*}{12.54} & \multirow{2}{*}{21.56} & \multirow{2}{*}{8.90} & \multirow{2}{*}{8.11} & \multirow{2}{*}{4.10} \\
\hline
\end{tabular}

\section{Results and discussion}

\section{Vegetative growth}

The results obtained in tables (2-7) showed that all growth characters including, plant height, number of tillers/plant, number of inflorescences/plant, inflorescences length, fresh and dry weight of herb were gradually decreased by increasing saline water levels In both seasons, the highest values of all these characters were obtained due to the use of the high saline water levels (4000). there reduction under higher salt concentrations, plants take up more toxic ions as compared to normal saline conditions and consequently the concentration of those ions becomes higher inside the tissues so the osmotic potential more negative and reduce the plant growth and development (Akhtar et al., 2013). Under saline conditions, water potential is disturbed which causes ionic imbalance that reduce the agricultural plants productivity. The reduction of plant growth actually depends on severity and levels of the salinity (Kausar et al., 2012). These results were agreed with those reported by Farahat, (2013) on Grevillea robusta plant, Amirijani, (2015) on periwinkle plants, Hashish et al., (2015) on gladiolus plants and Nahed et al., 2020 on Doranta erecta seedlings, they found that application of saline water alone led to significant reduction in all growth parameters.

Concerning the effect of potassium silicate on seedlings growth characters, data in Tables (27) mentioned that application of potassium silicate at $4 \mathrm{~g}$ rate had significant favorable effect on all previous parameters in the two seasons under irrigation with saline water at $2000 \mathrm{ppm}$. Treatment with the rate of $4 \mathrm{~g}$ potassium silicate, the increment was $10.20,0.50,0.98,0.39,0.36$ and $3.27 \%$ ) for plant height, tillers number/plant, inflorescences number/plant, inflorescence length, fresh and dry weight of herb/plant, in the first season respectively compared with the control plants. In the second season, the increase was $(0.22,0.59,1.06,0.39,0.31$ and $3.04 \%)$ for the same characters, respectively, compared with the untreated plants. The positive effect of potassium silicate on these parameters can be attributed to the role of $\mathrm{K}$ in regulating the opening and dosing of stomata and water retention. Also, K promotes the growth of meristematic tissues, activates some enzymatic reaction, aid in nitrogen metabolism and synthesis of proteins, catalyzes activities of some mineral elements, and aids in carbohydrates metabolism and translocation, Bhandal and Malik (1988). Also, this improvement may be due to the role of silicon in enhancing plant growth and promoting desirable plant physiological processes (Korndorfer and Lepsch, (2001). These results were in a harmony with those found by Hashish et al., (2014), Hussein and Muhammed (2017) and Hamdy et al., (2018). 
Middle East J. Agric. Res., 9(4): 934-942, 2020

Table 2: Effect of salinity levels, Potassium silicate, Carboxylic acid and their interaction on plant height (cm.) of Pennisetum setacum cv. Rubrum.

\begin{tabular}{|c|c|c|c|c|c|c|c|c|}
\hline \multirow{3}{*}{ Treats. } & \multicolumn{4}{|c|}{2000 ppm } & \multicolumn{4}{|c|}{4000 ppm } \\
\hline & \multicolumn{8}{|c|}{ First season } \\
\hline & Cont. & C.A. $1 \mathrm{~cm} / \mathrm{L}$ & C.A. $2 \mathrm{~cm} / \mathrm{L}$ & Mean & Cont. & C.A. $1 \mathrm{~cm} / \mathrm{L}$ & C.A. $2 \mathrm{~cm} / \mathrm{L}$ & Mean \\
\hline Cont. & $68.50 \mathrm{~g}$ & $71.23 \mathrm{f}$ & $72.55 \mathrm{f}$ & $70.76 \mathrm{C}$ & $58.31 \mathrm{~g}$ & $63.71 \mathrm{f}$ & $60.57 \mathrm{f}$ & $60.86 \mathrm{C}$ \\
\hline P. S. $2 \mathrm{~g}$ & $74.55 \mathrm{e}$ & $83.74 \mathrm{c}$ & $80.78 \mathrm{~d}$ & $79.69 \mathrm{~B}$ & $65.04 \mathrm{e}$ & $70.21 \mathrm{c}$ & $68.28 \mathrm{~d}$ & $67.84 \mathrm{~B}$ \\
\hline P.S. $4 \mathrm{~g}$ & $76.97 \mathrm{e}$ & $92.65 \mathrm{a}$ & $85.32 \mathrm{~b}$ & $84.98 \mathrm{~A}$ & $67.22 \mathrm{e}$ & $75.36 \mathrm{a}$ & $72.09 \mathrm{~b}$ & $71.55 \mathrm{~A}$ \\
\hline \multirow[t]{2}{*}{ Mean } & $73.34 \mathrm{C}$ & $82.54 \mathrm{~A}$ & $79.55 \mathrm{~B}$ & & 66.32 & 69.76 & 66.98 & \\
\hline & \multicolumn{8}{|c|}{ Second season } \\
\hline Cont. & $67.08 \mathrm{~g}$ & $72.08 \mathrm{f}$ & $73.22 \mathrm{f}$ & $70.79 \mathrm{C}$ & $55.87 \mathrm{~g}$ & $64.55 \mathrm{f}$ & $61.75 \mathrm{f}$ & $60.72 \mathrm{C}$ \\
\hline P. S. $2 \mathrm{~g}$ & $75.11 \mathrm{e}$ & $82.13 c$ & $79.91 \mathrm{~d}$ & $79.05 \mathrm{~B}$ & $66.14 \mathrm{e}$ & $71.36 \mathrm{c}$ & $68.03 \mathrm{~d}$ & $68.51 \mathrm{~B}$ \\
\hline P.S. $4 \mathrm{~g}$ & $76.05 \mathrm{e}$ & $95.02 \mathrm{a}$ & $87.32 \mathrm{~b}$ & $86.13 \mathrm{~A}$ & $68.09 \mathrm{e}$ & $74.97 \mathrm{a}$ & $71.12 \mathrm{~b}$ & $71.39 \mathrm{~A}$ \\
\hline Mean & $72.75 \mathrm{C}$ & $83.08 \mathrm{~A}$ & $80.15 \mathrm{~B}$ & & & & $66.97 \mathrm{~B}$ & \\
\hline
\end{tabular}

Table 3: Effect of salinity levels, Potassium silicate, Carboxylic acid and their interaction on No. of tillers /plant of Pennisetum setacum cv. Rubrum.

\begin{tabular}{|c|c|c|c|c|c|c|c|c|}
\hline \multirow{3}{*}{ Treats. } & \multicolumn{4}{|c|}{2000 ppm } & \multicolumn{4}{|c|}{4000 ppm } \\
\hline & \multicolumn{8}{|c|}{ First season } \\
\hline & Cont. & $\begin{array}{c}\text { C.A. } \\
1 \mathrm{~cm} / \mathrm{L}\end{array}$ & $\begin{array}{c}\text { C.A. } \\
2 \mathrm{~cm} / \mathrm{L}\end{array}$ & Mean & Cont. & $\begin{array}{c}\text { C.A. } \\
1 \mathrm{~cm} / \mathrm{L}\end{array}$ & $\begin{array}{c}\text { C.A. } \\
2 \mathrm{~cm} / \mathrm{L}\end{array}$ & Mean \\
\hline Cont. & $10.06 \mathrm{f}$ & $11.22 \mathrm{e}$ & $11.43 \mathrm{e}$ & $10.90 \mathrm{C}$ & $8.57 \mathrm{~g}$ & $9.27 \mathrm{f}$ & $9.32 \mathrm{f}$ & $9.05 \mathrm{C}$ \\
\hline P.S. $2 \mathrm{~g}$ & $12.72 \mathrm{~d}$ & $14.06 \mathrm{c}$ & $13.88 \mathrm{c}$ & $13.55 \mathrm{~B}$ & $11.43 \mathrm{e}$ & $13.58 \mathrm{c}$ & $12.51 \mathrm{~d}$ & $12.51 \mathrm{~B}$ \\
\hline P.S. $4 \mathrm{~g}$ & $12.81 \mathrm{~d}$ & $19.81 \mathrm{a}$ & $16.54 \mathrm{~b}$ & $16.39 \mathrm{~A}$ & $11.45 \mathrm{e}$ & $16.71 \mathrm{a}$ & $14.63 \mathrm{~b}$ & $14.26 \mathrm{~A}$ \\
\hline \multirow[t]{2}{*}{ Mean } & $11.86 \mathrm{C}$ & $15.03 \mathrm{~A}$ & $13.95 \mathrm{~B}$ & & $10.48 \mathrm{C}$ & $13.19 \mathrm{~A}$ & $12.15 \mathrm{~B}$ & \\
\hline & \multicolumn{8}{|c|}{ Second season } \\
\hline Cont. & $10.02 \mathrm{f}$ & $10.91 \mathrm{e}$ & $11.41 \mathrm{e}$ & 10.78 & $8.52 \mathrm{~h}$ & $9.30 \mathrm{f}$ & $9.37 \mathrm{~g}$ & $9.06 \mathrm{C}$ \\
\hline P.S. $2 \mathrm{~g}$ & $12.74 \mathrm{~d}$ & $15.11 \mathrm{c}$ & $14.86 \mathrm{c}$ & 14.24 & $11.39 \mathrm{e}$ & $13.60 \mathrm{c}$ & $11.53 \mathrm{~d}$ & $12.17 \mathrm{~B}$ \\
\hline P.S. $4 g$ & $12.87 \mathrm{~d}$ & $20.83 \mathrm{a}$ & $17.58 \mathrm{~b}$ & 17.09 & $11.44 \mathrm{e}$ & $16.75 \mathrm{a}$ & $14.76 \mathrm{~b}$ & $14.32 \mathrm{~A}$ \\
\hline Mean & $11.88 \mathrm{C}$ & $15.62 \mathrm{~A}$ & $14.62 \mathrm{~B}$ & & $10.45 \mathrm{C}$ & $13.22 \mathrm{~A}$ & $11.89 \mathrm{~B}$ & \\
\hline
\end{tabular}

Table 4: Effect of salinity levels, Potassium silicate, Carboxylic acid and their interaction on No. of inflorescences/ plant of Pennisetum setacum cv. Rubrum.

\begin{tabular}{|c|c|c|c|c|c|c|c|c|}
\hline \multirow{3}{*}{ Treats. } & \multicolumn{4}{|c|}{2000 ppm } & \multicolumn{4}{|c|}{4000 ppm } \\
\hline & \multicolumn{8}{|c|}{ First season } \\
\hline & Cont. & $\begin{array}{l}\text { C.A. } \\
1 \mathrm{~cm}\end{array}$ & $\begin{array}{l}\text { C.A. } \\
2 \mathrm{~cm}\end{array}$ & Mean & Cont. & $\begin{array}{l}\text { C.A. } \\
1 \mathrm{~cm} .\end{array}$ & $\begin{array}{l}\text { C.A. } \\
2 \mathrm{~cm}\end{array}$ & Mean \\
\hline Cont. & $12.65 \mathrm{f}$ & $14.82 \mathrm{e}$ & $15.54 \mathrm{e}$ & $14.34 \mathrm{C}$ & $7.05 \mathrm{e}$ & $8.14 \mathrm{de}$ & $8.87 \mathrm{~d}$ & $8.02 \mathrm{C}$ \\
\hline P. S. 2g & $19.09 \mathrm{~d}$ & $27.12 \mathrm{c}$ & $25.35 \mathrm{~cd}$ & $23.85 \mathrm{~B}$ & $9.66 \mathrm{~d}$ & $11.73 \mathrm{c}$ & $11.03 \mathrm{c}$ & $10.81 \mathrm{~B}$ \\
\hline P.S. 4g & $22.32 \mathrm{~d}$ & $33.56 \mathrm{a}$ & $29.17 \mathrm{~b}$ & $28.35 \mathrm{~A}$ & $10.08 \mathrm{~cd}$ & $15.75 \mathrm{a}$ & $12.43 \mathrm{~b}$ & $12.75 \mathrm{~A}$ \\
\hline \multirow[t]{2}{*}{ Mean } & $18.02 \mathrm{C}$ & $25.17 \mathrm{~A}$ & $23.35 \mathrm{~B}$ & & $8.93 \mathrm{C}$ & $11.87 \mathrm{~A}$ & $10.78 \mathrm{~B}$ & \\
\hline & \multicolumn{8}{|c|}{ Second season } \\
\hline Cont. & $11.89 \mathrm{f}$ & $15.11 \mathrm{e}$ & $14.87 \mathrm{e}$ & $13.96 \mathrm{C}$ & $6.88 \mathrm{e}$ & $8.22 \mathrm{de}$ & $9.07 \mathrm{~d}$ & $8.06 \mathrm{C}$ \\
\hline P.S. $2 \mathrm{~g}$ & $18.90 \mathrm{~d}$ & $27.58 \mathrm{c}$ & $25.05 \mathrm{~cd}$ & $23.84 \mathrm{~B}$ & $9.55 \mathrm{~d}$ & $12.69 \mathrm{c}$ & $12.08 \mathrm{c}$ & $11.44 \mathrm{~B}$ \\
\hline P.S. 4g & $20.92 \mathrm{~d}$ & $34.15 \mathrm{a}$ & $31.21 \mathrm{~b}$ & $28.76 \mathrm{~A}$ & $10.32 \mathrm{~d}$ & $16.05 \mathrm{a}$ & $13.34 \mathrm{~b}$ & $13.24 \mathrm{~A}$ \\
\hline Mean & $17.24 \mathrm{C}$ & $25.61 \mathrm{~A}$ & $23.71 \mathrm{~B}$ & & $8.92 \mathrm{C}$ & $12.32 \mathrm{~A}$ & $11.50 \mathrm{~B}$ & \\
\hline
\end{tabular}

Table 5: Effect of salinity levels, Potassium silicate, Carboxylic acid and their interaction on Inflorescence length (cm) of Pennisetum setacum cv. Rubrum.

\begin{tabular}{|c|c|c|c|c|c|c|c|c|}
\hline \multirow{3}{*}{ Treats. } & \multicolumn{4}{|c|}{2000 ppm } & \multicolumn{4}{|c|}{4000 ppm } \\
\hline & \multicolumn{8}{|c|}{ First season } \\
\hline & Cont. & $\begin{array}{l}\text { C.A. } \\
1 \mathrm{~cm} \\
\end{array}$ & $\begin{array}{l}\text { C.A. } \\
2 \mathrm{~cm}\end{array}$ & Mean & Cont. & $\begin{array}{l}\text { C.A. } \\
1 \mathrm{~cm} .\end{array}$ & $\begin{array}{l}\text { C.A. } \\
2 \mathrm{~cm}\end{array}$ & Mean \\
\hline Cont. & $17.11 \mathrm{f}$ & $20.05 \mathrm{e}$ & $19.12 \mathrm{e}$ & $18.76 \mathrm{C}$ & $13.25 \mathrm{f}$ & $14.32 \mathrm{e}$ & $15.02 \mathrm{e}$ & $14.20 \mathrm{C}$ \\
\hline P. S. $2 \mathrm{~g}$ & $21.08 \mathrm{~d}$ & $23.15 \mathrm{c}$ & $21.25 \mathrm{~d}$ & $21.83 \mathrm{~B}$ & $17.12 \mathrm{~d}$ & $18.72 \mathrm{c}$ & $17.27 \mathrm{~d}$ & $17.70 \mathrm{~B}$ \\
\hline P.S. $4 \mathrm{~g}$ & $22.45 \mathrm{~cd}$ & $30.36 \mathrm{a}$ & $25.21 \mathrm{~b}$ & $26.01 \mathrm{~A}$ & $18.45 \mathrm{c}$ & $23.11 \mathrm{a}$ & $20.07 \mathrm{~b}$ & $20.54 \mathrm{~A}$ \\
\hline \multirow[t]{2}{*}{ Mean } & $20.21 \mathrm{C}$ & $24.52 \mathrm{~A}$ & $21.86 \mathrm{~B}$ & & $16.27 \mathrm{~B}$ & $18.72 \mathrm{~A}$ & $17.65 \mathrm{~A}$ & \\
\hline & \multicolumn{8}{|c|}{ Second season } \\
\hline Cont. & $16.51 \mathrm{f}$ & $21.10 \mathrm{e}$ & $18.97 \mathrm{e}$ & $18.86 \mathrm{C}$ & $13.45 \mathrm{f}$ & $15.11 \mathrm{e}$ & $16.45 \mathrm{e}$ & $15.00 \mathrm{C}$ \\
\hline P. S. $2 \mathrm{~g}$ & $20.12 \mathrm{~d}$ & $23.33 \mathrm{c}$ & $22.41 \mathrm{~d}$ & $21.95 \mathrm{~B}$ & $17.51 \mathrm{~d}$ & $19.02 \mathrm{c}$ & $18.02 \mathrm{~d}$ & $18.18 \mathrm{~B}$ \\
\hline P. S. 4g & $22.22 \mathrm{~cd}$ & $31.54 \mathrm{a}$ & $25.08 \mathrm{~b}$ & $26.28 \mathrm{~A}$ & $18.52 \mathrm{c}$ & $22.30 \mathrm{a}$ & $20.33 \mathrm{~b}$ & $20.38 \mathrm{~A}$ \\
\hline Mean & $19.62 \mathrm{C}$ & $25.32 \mathrm{~A}$ & $22.15 \mathrm{~B}$ & & $16.49 \mathrm{~B}$ & $18.81 \mathrm{~A}$ & $18.27 \mathrm{~A}$ & \\
\hline
\end{tabular}


Middle East J. Agric. Res., 9(4): 934-942, 2020

Table 6: Effect of salinity levels, Potassium silicate, Carboxylic acid and their interaction on Herb fresh weight/plant (g) of Pennisetum setacum cv. Rubrum.

\begin{tabular}{|c|c|c|c|c|c|c|c|c|}
\hline \multirow{3}{*}{ Treats. } & \multicolumn{4}{|c|}{2000 ppm } & \multicolumn{4}{|c|}{4000 ppm } \\
\hline & \multicolumn{8}{|c|}{ First season } \\
\hline & Cont. & $\begin{array}{c}\text { C.A. } \\
1 \mathrm{~cm} / \mathrm{L}\end{array}$ & $\begin{array}{c}\text { C.A. } \\
2 \mathrm{~cm} / \mathrm{L}\end{array}$ & Mean & Cont. & $\begin{array}{c}\text { C.A. } \\
1 \mathrm{~cm} / \mathrm{L}\end{array}$ & $\begin{array}{c}\text { C.A. } \\
2 \mathrm{~cm} / \mathrm{L}\end{array}$ & Mean \\
\hline Cont. & $50.43 \mathrm{f}$ & $56.06 \mathrm{e}$ & $57.23 \mathrm{e}$ & $54.57 \mathrm{C}$ & $42.00 \mathrm{e}$ & $45.15 \mathrm{~d}$ & $46.78 \mathrm{~d}$ & $44.64 \mathrm{C}$ \\
\hline P.S. $2 \mathrm{~g}$ & $60.62 \mathrm{~d}$ & $64.17 \mathrm{c}$ & $63.12 \mathrm{c}$ & $62.64 \mathrm{~B}$ & $51.71 \mathrm{c}$ & $57.31 \mathrm{c}$ & $56.42 \mathrm{c}$ & $55.15 \mathrm{~B}$ \\
\hline P.S. 4g & $61.75 \mathrm{~d}$ & $77.55 \mathrm{a}$ & $71.26 \mathrm{~b}$ & $74.41 \mathrm{~A}$ & $52.07 \mathrm{c}$ & $62.84 \mathrm{a}$ & $59.21 \mathrm{~b}$ & $58.04 \mathrm{~A}$ \\
\hline \multirow[t]{2}{*}{ Mean } & $55.53 \mathrm{C}$ & $65.93 \mathrm{~A}$ & $63.87 \mathrm{~B}$ & & $48.59 \mathrm{C}$ & $55.10 \mathrm{~A}$ & $54.14 \mathrm{~B}$ & \\
\hline & \multicolumn{8}{|c|}{ Second season } \\
\hline Cont. & $49.55 \mathrm{f}$ & $55.60 \mathrm{e}$ & $57.08 \mathrm{e}$ & 54.08 & $40.94 \mathrm{e}$ & $44.08 \mathrm{~d}$ & $46.44 \mathrm{~d}$ & $43.82 \mathrm{C}$ \\
\hline P.S. $2 \mathrm{~g}$ & $60.21 \mathrm{~d}$ & $65.88 \mathrm{c}$ & $64.27 \mathrm{c}$ & 63.45 & $50.12 \mathrm{c}$ & $56.47 \mathrm{c}$ & $57.01 \mathrm{c}$ & $54.53 \mathrm{~B}$ \\
\hline P.S. 4g & $60.81 \mathrm{~d}$ & $79.98 \mathrm{a}$ & $71.67 \mathrm{~b}$ & 70.82 & $51.90 \mathrm{c}$ & $63.52 \mathrm{a}$ & $59.38 \mathrm{~b}$ & $58.27 \mathrm{~A}$ \\
\hline Mean & $56.86 \mathrm{C}$ & $67.15 \mathrm{~A}$ & $64.34 \mathrm{~B}$ & & $47.65 \mathrm{C}$ & $54.69 \mathrm{~A}$ & $54.28 \mathrm{~B}$ & \\
\hline
\end{tabular}

Table 7: Effect of salinity levels, Potassium silicate, Carboxylic acid and their interaction on Herb dry weight/plant ( $\mathrm{g}$ ) of Pennisetum setacum cv. Rubrum.

\begin{tabular}{|c|c|c|c|c|c|c|c|c|}
\hline \multirow{3}{*}{ Treats. } & \multicolumn{4}{|c|}{2000 ppm } & \multicolumn{4}{|c|}{4000 ppm } \\
\hline & \multicolumn{8}{|c|}{ First season } \\
\hline & Cont. & $\begin{array}{c}\text { C.A. } \\
1 \mathrm{~cm} / \mathrm{L}\end{array}$ & $\begin{array}{c}\text { C.A. } \\
2 \mathrm{~cm} / \mathrm{L}\end{array}$ & Mean & Cont. & $\begin{array}{c}\text { C.A. } \\
1 \mathrm{~cm} / \mathrm{L}\end{array}$ & $\begin{array}{c}\text { C.A. } \\
2 \mathrm{~cm} / \mathrm{L}\end{array}$ & Mean \\
\hline Cont. & $1.15 \mathrm{f}$ & $5.12 \mathrm{e}$ & $6.32 \mathrm{e}$ & $4.20 \mathrm{C}$ & $0.12 \mathrm{f}$ & $0.25 \mathrm{e}$ & $0.29 \mathrm{e}$ & $0.22 \mathrm{C}$ \\
\hline P.S. $2 \mathrm{~g}$ & $8.87 \mathrm{~d}$ & $12.58 \mathrm{c}$ & $11.55 \mathrm{c}$ & $11.00 \mathrm{~B}$ & $1.02 \mathrm{~d}$ & $5.65 \mathrm{c}$ & $4.09 \mathrm{c}$ & $3.59 \mathrm{~B}$ \\
\hline P.S. $4 g$ & $9.33 \mathrm{~d}$ & $25.41 \mathrm{a}$ & $19.05 \mathrm{~b}$ & $17.93 \mathrm{~A}$ & $1.84 \mathrm{~d}$ & $10.22 \mathrm{a}$ & $7.54 \mathrm{~b}$ & $6.53 \mathrm{~A}$ \\
\hline \multirow[t]{2}{*}{ Mean } & $6.45 \mathrm{C}$ & $14.37 \mathrm{~A}$ & $12.31 \mathrm{~B}$ & & $0.99 \mathrm{C}$ & $5.37 \mathrm{~A}$ & $3.97 \mathrm{~B}$ & \\
\hline & \multicolumn{8}{|c|}{ Second season } \\
\hline Cont. & $1.20 \mathrm{f}$ & $6.05 \mathrm{e}$ & $6.84 \mathrm{e}$ & $4.70 \mathrm{C}$ & $0.19 \mathrm{f}$ & $0.31 \mathrm{e}$ & $0.29 \mathrm{e}$ & $0.26 \mathrm{C}$ \\
\hline P.S. $2 \mathrm{~g}$ & $8.56 \mathrm{~d}$ & $13.11 \mathrm{c}$ & $12.05 \mathrm{c}$ & $11.24 \mathrm{~B}$ & $1.22 \mathrm{~d}$ & $5.87 \mathrm{c}$ & $5.10 \mathrm{c}$ & $4.06 \mathrm{~B}$ \\
\hline P.S. $4 g$ & $9.54 \mathrm{~d}$ & $27.19 \mathrm{a}$ & $20.20 \mathrm{~b}$ & $18.98 \mathrm{~A}$ & $2.08 \mathrm{~d}$ & $11.18 \mathrm{a}$ & $8.01 \mathrm{~b}$ & $7.09 \mathrm{~A}$ \\
\hline Mean & $6.43 \mathrm{C}$ & $15.45 \mathrm{~A}$ & $13.03 \mathrm{~B}$ & & $1.16 \mathrm{C}$ & $5.79 \mathrm{~A}$ & $4.47 \mathrm{~B}$ & \\
\hline
\end{tabular}

Regarding the effect of carboxylic acid on plant growth parameters of Pennisetum setacum, the data in the same tables revealed that all previous growth characters significantly affected by carboxylic acid application at 1 and $2 \mathrm{~cm} / \mathrm{L}$ compared to control plants under irrigation with saline water at $2000 \mathrm{ppm}$. Spraying plants with $1 \mathrm{~cm} / \mathrm{L}$ gave the highest values in all growth parameters. Numerically, plant height, number of number of tillers/plant, number of inflorescences/plant, inflorescences length, fresh and dry weight of herb/plant were increased by $(0.13,0.27,0.40,0.21$, 0.19 and $1.23 \%$ ), respectively, in comparison with the control plant in the first season. In this respect, in the second season such increments were estimated by $0.14,0.31,0.49,0.29,0.18$ and $1.40 \%$ compared with untreated plants, respectively. This may demonstrate that carboxylic in different concentration could be used to increase morphological parameters in some plants (Castro et al., 2003) these results were confirmed by William and Jeiry, (2017); Khaled (2017) and Ishelmbule et al., (2018).

As regard the interaction between potassium silicate and carboxylic acid under irrigation with saline water at two levels (2000ppm and 4000ppm), data presented in Tables (2-7) indicated that, the interaction between different factors (potassium silicate and carboxylic acid) under salinity at (2000 or 4000 ppm) was almost significant for vegetative growth characters in both seasons. The highest value due to the previous factors were obtained to potassium silicate at $2 \mathrm{~g} *$ carboxylic acid at $1 \mathrm{~cm} / \mathrm{L}$ under salinity at 2000ppm in both seasons. the highest values obtained were $(92.65$ and 95.02) for plant height, (19.81 and 20.83) for tillers numbers, (33.56 and 34.15) for inflorescences number, (30.36 and 31.54) for inflorescences length, (77.55 and 79.98) for herb fresh weight and (25.41 and 27.19) for herb dry weight.

\section{Chemical constituents}

\subsection{Carbohydrates \%}

Data on the effect of different salinity levels and various potassium silicate, carboxylic acid and their interaction on total carbohydrates percentage of Pennisetum setacum in leaves are presented in Table (8). It is clear from data that raising the level of salinity markedly decreased the 
total carbohydrates. Percentage from 2000 and 4000ppm in both seasons. The reduction in total carbohydrates as salinity levels increased may be related to respiration processes since the free sugar was the main sugar pattern involve in the mechanism of respiration (Bernstein et al., 1972). The present finding are generally in accordance with those reported by Eid et al., (2011) on Tagets erecta, Mazhar et al. (2012) on Chrysanthemum indicum and Farahat et al. (2013) on Gerevilla robasta plant. From the given data in the same table, it can be concluded that, increasing potassium silicate rates caused an increase in the content of total carbohydrates percentage compared with the control in both seasons. The highest value was obtained by potassium silicate at the rate of $4 \mathrm{~g}$ were $(41.75$ and $44.12 \%)$ in the first and second seasons respectively, under irrigation with salinity at $2000 \mathrm{ppm}$. In the context, carboxylic acid increased total carbohydrates percentage in both seasons. Carboxylic acid at the rate of $1 \mathrm{~cm} / \mathrm{L}$ were (39.59 and 42.10\%) respectively, under saline water at $2000 \mathrm{ppm}$, in the first and second seasons. Concerning interaction between potassium silicate at $4 \mathrm{gm}+$ carboxylic acid at $1 \mathrm{~cm} / \mathrm{L}$ gave the highest values of total carbohydrates percentage were (35.41 and $49.70 \%)$ in the first and second seasons, respectively, under saline water at 2000ppm.

Table 8: Effect of salinity levels, Potassium silicate, Carboxylic acid and their interaction on Total carbohydrates of Pennisetum setacum cv. Rubrum.

\begin{tabular}{|c|c|c|c|c|c|c|c|c|}
\hline \multirow{3}{*}{ Treats. } & \multicolumn{4}{|c|}{2000 ppm } & \multicolumn{4}{|c|}{4000 ppm } \\
\hline & \multicolumn{8}{|c|}{ First season } \\
\hline & Cont. & $\begin{array}{c}\text { C.A. } \\
1 \mathrm{~cm} / \mathrm{L}\end{array}$ & $\begin{array}{c}\text { C.A. } \\
2 \mathrm{~cm} / \mathrm{L}\end{array}$ & Mean & Cont. & $\begin{array}{c}\text { C.A. } \\
1 \mathrm{~cm} / \mathrm{L}\end{array}$ & $\begin{array}{c}\text { C.A. } \\
2 \mathrm{~cm} / \mathrm{L}\end{array}$ & Mean \\
\hline Cont. & $30.05 \mathrm{f}$ & $33.12 \mathrm{e}$ & $33.98 \mathrm{e}$ & $32.38 \mathrm{C}$ & $20.11 \mathrm{f}$ & $22.36 \mathrm{e}$ & $23.08 \mathrm{e}$ & $21.85 \mathrm{C}$ \\
\hline P.S. $2 \mathrm{~g}$ & $36.2 \mathrm{~d}$ & $40.23 \mathrm{c}$ & $39.01 \mathrm{c}$ & $38.48 \mathrm{~B}$ & $25.36 \mathrm{~d}$ & $31.02 \mathrm{c}$ & $29.21 \mathrm{c}$ & $28.53 \mathrm{~B}$ \\
\hline P. S. 4g & $37.54 \mathrm{~d}$ & $45.41 \mathrm{a}$ & $42.31 b$ & $41.75 \mathrm{~A}$ & $27.47 \mathrm{~d}$ & $36.55 \mathrm{a}$ & $33.05 \mathrm{~b}$ & $32.36 \mathrm{~A}$ \\
\hline \multirow[t]{2}{*}{ Mean } & $34.60 \mathrm{C}$ & $39.59 \mathrm{~A}$ & $38.43 \mathrm{~B}$ & & $24.31 \mathrm{~B}$ & $29.98 \mathrm{~A}$ & $28.45 \mathrm{~A}$ & \\
\hline & \multicolumn{8}{|c|}{ Second season } \\
\hline Cont. & $33.21 \mathrm{f}$ & $35.5 \mathrm{e}$ & $36.17 \mathrm{e}$ & $34.96 \mathrm{C}$ & $22.04 \mathrm{f}$ & $23.1 \mathrm{e}$ & $23.56 \mathrm{e}$ & $22.90 \mathrm{C}$ \\
\hline P.S. 2g & $37.03 \mathrm{~d}$ & $41.09 \mathrm{c}$ & $39.02 \mathrm{c}$ & $39.05 \mathrm{~B}$ & $25.93 \mathrm{~d}$ & $33.44 \mathrm{c}$ & $30.25 \mathrm{c}$ & $29.87 \mathrm{~B}$ \\
\hline P. S. 4g & $38.55 \mathrm{~d}$ & $49.7 \mathrm{a}$ & $44.1 \mathrm{~b}$ & $44.12 \mathrm{~A}$ & $28.06 \mathrm{~d}$ & $40.2 \mathrm{a}$ & $36.32 \mathrm{~b}$ & $34.86 \mathrm{~A}$ \\
\hline Mean & $36.26 \mathrm{C}$ & $42.10 \mathrm{~A}$ & $39.76 \mathrm{~B}$ & & $25.34 \mathrm{~B}$ & $32.25 \mathrm{~A}$ & $30.04 \mathrm{~A}$ & \\
\hline
\end{tabular}

\subsection{Nutrients}

Data of nutrients (N, P and $\mathrm{K}$ ) in leaves as affected by different salinity levels and micro fertilizers application treatments in both seasons throughout experimental periods are presented in Tables (9-11). It is clear from data that increasing salinity levels reduced the percentage of N, P and $\mathrm{K}$ in the two growing seasons. In this connection, Hanafy, (1996) pointed out that salinization impaired $\mathrm{N}$ accumulation and incorporation into protein and raised total free amino acid accumulation in salinized plants. Also, it can be suggested that amino acid can act as components of salt tolerance mechanism and build up a favorable osmotic potential inside the cell in order to combat the effects of which replaced nitration the vacuoles. Furthermore, the reduction in $\mathrm{P}$ up take under saline condition could be explained on the fact that $\mathrm{Na}$ salt raised the $\mathrm{pH}$ of the soil, which in turn reduced the availability of P to the plants (Sonneveld and Voegt, 1983). In this context, Gamed et al. (1996) found an increase in $\mathrm{Na}$ concentration and decrease in $\mathrm{K}$ concentration in the leaves with salinity, this result may be due to a possible antagonism between $\mathrm{K}$ and $\mathrm{Na}$. this antagonism could be due to direct competition between $\mathrm{K}$ and $\mathrm{Na}$ at the site of ion uptake at plasma lemma. In close agreement with these result were the finding respected on Sesbania aegyptiaca (Azza et al., 2006), Khaya senegalensis (Nahed et al., 2006) and Grevilla robusta (Farahat et al., 2013).As regarding the effect of potassium silicate and carboxylic acid and $\mathrm{N}, \mathrm{P}$ and $\mathrm{K}$ concentration, it is to be noticed from the results that, the percentage of $\mathrm{N}, \mathrm{P}$ and $\mathrm{K}$ increased by treating plants with the previous application treatments compared with the untreated plants. It was shown that, $\mathrm{N}, \mathrm{P}$ and $\mathrm{K}$ percentage gradually increased by increasing potassium silicate levels (4g), the highest values was (1.45 and $1.49 \%) \mathrm{N},(0.23$ and $0.28 \%) \mathrm{P}$ and (1.52 and $1.55 \%) \mathrm{P}$ in both seasons respectively, under irrigation with saline water at 2000ppm. Also, carboxylic acid at $1 \mathrm{~cm} / \mathrm{L}$ resulted in the highest percentage of $\mathrm{N}, \mathrm{P}$ and $\mathrm{K}$ which gave $(1.40$ and $1.43 \%) \mathrm{N},(0.23$ and $0.26 \%)$ and $(1.48$ and $1.50 \%) \mathrm{K}$ in the first and second seasons respectively, under salinity at $2000 \mathrm{ppm}$. 
Concerning, the interaction between potassium silicate and carboxylic acid data in Tables (911) revealed that, the application of potassium silicate at $4 \mathrm{~g}$ and carboxylic acid at $1 \mathrm{~cm} / \mathrm{L}$ gave the highest values of the previous nutrients in comparison with the control plant in both seasons under irrigation with saline water at $2000 \mathrm{ppm}$.

Table 9: Effect of salinity levels, Potassium silicate, Carboxylic acid and their interaction on N\% of Pennisetum setacum cv. Rubrum.

\begin{tabular}{|c|c|c|c|c|c|c|c|c|}
\hline \multirow[t]{3}{*}{ Treats. } & \multicolumn{5}{|c|}{2000 ppm } & \multicolumn{2}{|c|}{$4000 \mathrm{ppm}$} & \multirow[b]{3}{*}{ Mean } \\
\hline & \multicolumn{7}{|c|}{ First season } & \\
\hline & Cont. & $\begin{array}{c}\text { C.A. } \\
1 \mathrm{~cm} / \mathrm{L}\end{array}$ & $\begin{array}{c}\text { C.A. } \\
2 \mathrm{~cm} / \mathrm{L}\end{array}$ & Mean & Cont. & $\begin{array}{c}\text { C.A. } \\
1 \mathrm{~cm} / \mathrm{L}\end{array}$ & $\begin{array}{c}\text { C.A. } \\
2 \mathrm{~cm} / \mathrm{L}\end{array}$ & \\
\hline Cont. & $1.23 \mathrm{f}$ & $1.26 \mathrm{e}$ & $1.27 \mathrm{e}$ & $1.25 \mathrm{C}$ & $0.61 \mathrm{~h}$ & $0.66 \mathrm{~g}$ & $0.70 \mathrm{f}$ & $0.66 \mathrm{C}$ \\
\hline P. S. $2 \mathrm{~g}$ & $1.30 \mathrm{~d}$ & $1.40 \mathrm{c}$ & $1.37 \mathrm{c}$ & $1.36 \mathrm{~B}$ & $0.75 \mathrm{e}$ & $0.89 \mathrm{c}$ & $0.85 \mathrm{~d}$ & $0.83 \mathrm{~B}$ \\
\hline P. S. 4g & $1.33 \mathrm{~d}$ & $1.55 \mathrm{a}$ & $1.48 \mathrm{~b}$ & $1.45 \mathrm{~A}$ & $0.78 \mathrm{e}$ & $1.13 \mathrm{a}$ & $1.02 \mathrm{~b}$ & $0.98 \mathrm{~A}$ \\
\hline Mean & $1.29 \mathrm{C}$ & $1.40 \mathrm{~A}$ & $1.37 \mathrm{~B}$ & & $0.71 \mathrm{C}$ & $0.89 \mathrm{~A}$ & $0.86 \mathrm{~B}$ & \\
\hline & \multicolumn{8}{|c|}{ Second season } \\
\hline Cont. & $1.25 \mathrm{f}$ & $1.28 \mathrm{e}$ & $1.28 \mathrm{e}$ & $1.27 \mathrm{C}$ & $0.65 \mathrm{~g}$ & $0.70 \mathrm{f}$ & $0.73 \mathrm{f}$ & $0.69 \mathrm{C}$ \\
\hline P. S. $2 \mathrm{~g}$ & $1.32 \mathrm{~d}$ & $1.41 \mathrm{c}$ & $1.38 \mathrm{c}$ & $1.37 \mathrm{~B}$ & $0.77 \mathrm{e}$ & $0.90 \mathrm{c}$ & $0.85 \mathrm{~d}$ & $0.84 \mathrm{~B}$ \\
\hline P.S. 4g & $1.35 \mathrm{~d}$ & $1.60 \mathrm{a}$ & $1.52 \mathrm{~b}$ & $1.49 \mathrm{~A}$ & $0.80 \mathrm{e}$ & $1.20 \mathrm{a}$ & $1.05 \mathrm{~b}$ & $1.02 \mathrm{~A}$ \\
\hline Mean & $1.31 \mathrm{C}$ & $1.43 \mathrm{~A}$ & $1.39 \mathrm{~B}$ & & $0.74 \mathrm{C}$ & $0.93 \mathrm{~A}$ & $0.87 \mathrm{~B}$ & \\
\hline
\end{tabular}

Table 10: Effect of salinity levels, Potassium silicate, Carboxylic acid and their interaction on $\mathrm{P} \%$ of Pennisetum setacum cv. Rubrum.

\begin{tabular}{|c|c|c|c|c|c|c|c|c|}
\hline \multirow{3}{*}{ Treats. } & \multicolumn{4}{|c|}{2000 ppm } & \multicolumn{4}{|c|}{4000 ppm } \\
\hline & \multicolumn{8}{|c|}{ First season } \\
\hline & Cont. & $\begin{array}{c}\text { C.A. } \\
1 \mathrm{~cm} / \mathrm{L}\end{array}$ & $\begin{array}{c}\text { C.A. } \\
2 \mathrm{~cm} / \mathrm{L}\end{array}$ & Mean & Cont. & $\begin{array}{c}\text { C.A. } \\
1 \mathrm{~cm} / \mathrm{L}\end{array}$ & $\begin{array}{c}\text { C.A. } \\
2 \mathrm{~cm} / \mathrm{L}\end{array}$ & Mean \\
\hline Cont. & $0.12 \mathrm{e}$ & $0.18 \mathrm{~d}$ & $0.18 \mathrm{~d}$ & 0.16 & $0.05 \mathrm{e}$ & $0.07 \mathrm{~d}$ & $0.07 \mathrm{~d}$ & $0.06 \mathrm{C}$ \\
\hline P.S. $2 \mathrm{~g}$ & $0.2 \mathrm{c}$ & $0.23 \mathrm{bc}$ & $0.23 \mathrm{bc}$ & 0.22 & $0.08 \mathrm{~cd}$ & $0.09 \mathrm{c}$ & $0.09 \mathrm{c}$ & $0.09 \mathrm{~B}$ \\
\hline P.S. $4 \mathrm{~g}$ & $0.21 \mathrm{c}$ & $0.27 \mathrm{a}$ & $0.24 \mathrm{~b}$ & 0.23 & $0.08 \mathrm{~cd}$ & $0.15 \mathrm{a}$ & $0.11 \mathrm{~b}$ & $0.11 \mathrm{~A}$ \\
\hline \multirow[t]{2}{*}{ Mean } & $0.18 \mathrm{~B}$ & $0.23 \mathrm{~A}$ & $0.22 \mathrm{~A}$ & & $0.07 \mathrm{~B}$ & $0.10 \mathrm{~A}$ & $0.09 \mathrm{~A}$ & \\
\hline & \multicolumn{8}{|c|}{ Second season } \\
\hline Cont. & $0.13 \mathrm{e}$ & $0.2 \mathrm{~d}$ & $0.21 \mathrm{~cd}$ & $0.18 \mathrm{C}$ & $0.06 \mathrm{e}$ & $0.08 \mathrm{~cd}$ & $0.08 \mathrm{~cd}$ & $0.07 \mathrm{C}$ \\
\hline P.S. $2 \mathrm{~g}$ & $0.22 \mathrm{~cd}$ & $0.24 \mathrm{c}$ & $0.23 \mathrm{c}$ & $0.23 \mathrm{~B}$ & $0.09 \mathrm{c}$ & $0.09 \mathrm{c}$ & $0.1 \mathrm{c}$ & $0.09 \mathrm{~B}$ \\
\hline P.S. 4g & $0.24 \mathrm{c}$ & $0.33 \mathrm{a}$ & $0.28 \mathrm{~b}$ & $0.28 \mathrm{~A}$ & $0.09 \mathrm{c}$ & $0.18 \mathrm{a}$ & $0.13 \mathrm{~b}$ & $0.13 \mathrm{~A}$ \\
\hline Mean & $0.20 \mathrm{~B}$ & $0.26 \mathrm{~A}$ & $0.24 \mathrm{AB}$ & & $0.08 \mathrm{~B}$ & $0.12 \mathrm{~A}$ & $0.10 \mathrm{AB}$ & \\
\hline
\end{tabular}

Table 11: Effect of salinity levels, Potassium silicate, Carboxylic acid and their interaction on $\mathrm{K} \%$ of Pennisetum setacum cv. Rubrum.

\begin{tabular}{|c|c|c|c|c|c|c|c|c|}
\hline \multirow{3}{*}{ Treats. } & \multicolumn{4}{|c|}{2000 ppm } & \multicolumn{4}{|c|}{4000 ppm } \\
\hline & \multicolumn{8}{|c|}{ First season } \\
\hline & Cont. & $\begin{array}{c}\text { C.A. } \\
1 \mathrm{~cm} / \mathrm{L}\end{array}$ & $\begin{array}{c}\text { C.A. } \\
2 \mathrm{~cm} / \mathrm{L}\end{array}$ & Mean & Cont. & $\begin{array}{c}\text { C.A. } \\
1 \mathrm{~cm} / \mathrm{L}\end{array}$ & $\begin{array}{c}\text { C.A. } \\
2 \mathrm{~cm} / \mathrm{L}\end{array}$ & Mean \\
\hline Cont. & $1.09 \mathrm{~g}$ & $1.22 \mathrm{f}$ & $1.20 \mathrm{f}$ & $1.17 \mathrm{C}$ & $0.85 \mathrm{~h}$ & $0.91 \mathrm{~g}$ & $0.92 \mathrm{~g}$ & $0.89 \mathrm{C}$ \\
\hline P.S. $2 \mathrm{~g}$ & $1.30 \mathrm{e}$ & $1.56 \mathrm{c}$ & $1.45 \mathrm{~d}$ & $1.44 \mathrm{~B}$ & $0.99 \mathrm{f}$ & $1.10 \mathrm{c}$ & $1.06 \mathrm{~d}$ & $1.05 \mathrm{~B}$ \\
\hline P.S. $4 \mathrm{~g}$ & $1.32 \mathrm{e}$ & $1.65 \mathrm{a}$ & $1.60 \mathrm{~b}$ & $1.52 \mathrm{~A}$ & $1.03 \mathrm{e}$ & $1.21 \mathrm{a}$ & $1.14 \mathrm{~b}$ & $1.13 \mathrm{~A}$ \\
\hline \multirow[t]{2}{*}{ Mean } & $1.24 \mathrm{C}$ & $1.48 \mathrm{~A}$ & $1.42 \mathrm{~B}$ & & $0.96 \mathrm{C}$ & $1.07 \mathrm{~A}$ & $1.04 \mathrm{~B}$ & \\
\hline & \multicolumn{8}{|c|}{ Second season } \\
\hline Cont. & $1.11 \mathrm{~g}$ & $1.24 \mathrm{f}$ & $1.23 \mathrm{f}$ & $1.19 \mathrm{C}$ & $0.88 \mathrm{~h}$ & $0.95 \mathrm{~g}$ & $0.96 \mathrm{~g}$ & $0.93 \mathrm{C}$ \\
\hline P.S. $2 \mathrm{~g}$ & $1.33 \mathrm{e}$ & $1.58 \mathrm{c}$ & $1.48 \mathrm{~d}$ & $1.46 \mathrm{~B}$ & $1.02 \mathrm{f}$ & $1.13 \mathrm{c}$ & $1.09 \mathrm{~d}$ & $1.08 \mathrm{~B}$ \\
\hline P.S. 4g & $1.35 \mathrm{e}$ & $1.68 \mathrm{a}$ & $1.62 \mathrm{~b}$ & $1.55 \mathrm{~A}$ & $1.08 \mathrm{e}$ & $1.25 \mathrm{a}$ & $1.18 \mathrm{~b}$ & $1.17 \mathrm{~A}$ \\
\hline Mean & $1.26 \mathrm{C}$ & $1.50 \mathrm{~A}$ & $1.44 \mathrm{~B}$ & & $0.99 \mathrm{C}$ & $1.11 \mathrm{~A}$ & $1.08 \mathrm{~B}$ & \\
\hline
\end{tabular}

\section{References}

A.O.A.C., 1995.Official Methods of Analysis Pub. By official A.O.A.C chapter 4, p.18-37, p.10, 44 p. 8-9.

Akhtar, M.S., J. Panwar and Y. Yeoung-Sang, 2013. Biogenic Synthesis of Metallic Nanoparticles by Plant Extracts. ACS Sustainable Chem. Eng., 1, 591-602.

Amal, A., B.E.L. Mohamed and S. Ewald, 2007. Response of crops to salinity under Egyptian conditions: a review. Landbauforschung Völkenrode, 2 (57):119-125. 
Amirijani, M. R., 2015. Effect of salinity stress on seed germination and antioxidative defense system of Catharanthus roseus. Journal of Agricultural and Biological Science., 5 (10): 163171.

Azza, M.; A. M.; Rawya A. Eid and Nahed G. Abd El- Aziz, 2006. Effect of Microbien under salt stress on nodulation, growth and chemical constituents of Sesbania aegyptiaca in sandy soil, Bull. NRC, Egypt, 31:247-268.

Bernstein, I., I.E. Francois and R.A. Clark, 1972. Salt tolerance of ornamental shrubs and ground covers. J. Amer. Soc. Hort. Sci., 97:550-556.

Bhandal, I. S. and Malik, C. P. (1988). Potassium estimation, uptake, and its role in the physiology and metabolism of flowering plants. AGRIS., (110), 205-254 pp.

Cabin, R.J., S.G. Weller, D.H. Lorence, T.W. Flynn, A.K. Sakai, D.R. Sandquist, and L.J. Hadway, 2000. Effects of long-term ungulate exclusion and recent alien species control on the preservation of and restoration of a Hawaiian tropical dry forest. Conservation Biology14: 439-453.

Cottenie, A., M. Verloo, L. Kiekens, G. Velghe, and R. Camerlynck, 1982. Chemical Analysis of Plant and Soil. Laboratory of Analytic and Agrochemistry, state Univ. Ghent Belgium, 100129.

Devis, J. and F. Freitas, 1970. Physical and Chemical Methods of Soil and Water Analysis. Soil Bulletin No. 10 Food and Agricultural Orgnization (FAO) Rome.

Dormaar, J. F., 1982. Aliphatic carboxylic acids in chernozemic soils. Can. J. Soil Sci., 62: 487494.

Edward, F.G., 1999. Pennisetumsetaceum 'Rubrum' Purple Fountain Grass. Cooperative Extension Service.Institute of Food and Agricultural Sciences. Fact Sheet FPS-464.

Eid, Rawia, A.,Taha Lobna S. and Ibrahiem, Soad M.M. 2011. Alleviation of adverse effect of salinity on growth, and chemical constituents of Marigold plants by using glutathione and ascorbate. J. of Appli. Sci. Res., 7(5): 714-721.

Epstein, E. and A.J. Bloom, 2005. Mineral Nutrition of Plants: Principles and Perspectives, 2nd ed. Sinauer Associates, Sunderland, MA.

Farahat, M.M., Azza, A.M., H. Mona, and M. Sahar, 2013. Salt tolerance in Grevillea robusta seedlings via foliar application of ascorbic acid. Middle East J. of Sci. Res., 14(1): 09-15.

Gold Book, 2014. International Union of Pure and Applied Chemistry, Compendium of Chemical Terminology. Version, 2.3.3.

Hamdy, E. B. M., S.A. Samira and S.M. Shahin, 2018. Effect of foliar spray with potassium silicate on growth and active constituents of horseradish armiractetra (Moringa oleifera Lam.) plants grown in some soils of Egypt. Middle East Journal of Agriculture., 7 (1): 60-70pp.

Hanafy, A.A.H., 1996. Physiological studies on tiploun and nitrate accumulation in lettuce plants. J. pf Agric. Sci., Mansoura Univ., 21: 3971-3994.

Hashish, Kh. I., Rawia Eid A., Magda M. Kandil and Azza A. M. Mazher (2015). Study on various level of salinity on some morphological and chemical composition of gladiolus plants by foliar spray with glutathione and thiamine. International Journal of Chem Tech Research 9(8): 334-341.

Hashish, Kh. I., E.F. Abdalla, M. M. Azza and T. A. El-Masiry, 2014. Influence of potassium foliar application on growth and chemical constituents of Jatropha curcas seedlings grown under water regime. International Journal of Academic Research, 6(2): 142-147.

Hussein, W.A. and M.M. Muhammed, 2017.The response of white eggplant plants to foliar application with boron and potassium silicate. Assiut J. Agric. Sci., 48: 394-401.

Kausar, H., S. Shazia, M.A. Muhammad and S. Abdus, 2012. Studies on the development and storage stability of cucumber-melon functional drink. J. Agric. Res., 50(2) 239

Khaled A.E., 2017. Effect of carboxylic acids fertilization on growth and chemical composition of Althaea rosea Cav. grown in different soil types. Middle East J. Agric. Res., 6(4): 1313-1327. 
Korndorfer, G.H. and I. Lepsch, 2001.Effect of silicon on plant growth and crop yield. Studies in Plant Science, 8: 133-147.

Lunatanab, F.S. and S.R. Olsen (1965). Test of an ascorbic acid method for determining phosphorus in water and $\mathrm{NaHCO}_{3}$ extracts from soil. Soil Sci. Soc. Arupr. Proc., 29: 677-678.

Marschner, H., 1995. Mineral Nutrition of Higher Plants, Academic Press, 417-426,440-442.

Mazhar, Azza, A.M., Shedeed, Shaymaa I., Abdel-Aziz, Nahed G. and Mahgoub, Mona, H., 2012. Growth, flowering and chemical constituents of Chrysanthemum indicum L. plant in response to different levels of humic acid and salinity. J. of Appli. Sci. Res. 8(7): 3697-3706.

Milton, S.J., J.H. Hoffmann, R.C.K. Bowie, J. D'Amico, M. Griffiths, D. Joubert, D. Loewenthal, N.N. Moinde, C. Seymour, M.V. Toral-Granda, and R. Wiseman, 1998. Invasive fountain grass on the Cape Peninsula. South African Journal of Science 94: 57-59.

Nahed, Abd El-Aziz, G., Azza A. M. Mazher and E. EL-Habba, 2006. Effect of foliar spraying with ascorbic acid on growth and chemical constituents of Khaya senegalensis grown under salt condition. American - Eurasian J. Agric. and Environ. Sci., 1 (3): 207-214.

Nahed, G. Abdel-Aziz, Azza, A. Mazher, Mona H. Mahgub, Mona A. Darwish, Rania M. A. Nassar and Ahmed S. Abdel-Aal, 2020. Effect of salinity stress on growth, chemical constituents and stem anatomy of Duranta erecta L. plants. Middle East Journal of Agriculture Research., 4 (9) 711-720 pp.

Pregel, F., 1945. Quantitative Organic Micro-Analysis. $4^{\text {th }}$ Ed, J. and A. Churchill, London, p. 203209.

SAS institute, 1994. SAS/STAT User s Guide: Statistics Vers. 604, $4^{\text {th }}$ Ed, SAS Institute. Inc., Cary, N.C., USA.

Smith, E.; M.A. Gilles; D.K. Hamilton and P.A. Gedees (1956).Colorimetric method for determination of sugars and related substances Anal. Chem., 28: 350.

Snedecor, G.W. and W.G. Cochran, 1980. Statistical Methods. 7th Edition, Iowa State University Press, Ames.

Sonneveld, C. I. and W. Voegt, 1983. Studies on the salt tolerance of some flower crops grown under glass. Plant and Soil., 74: 41-52.

Wang, S.Y. and G.J. Galletta, 1998. Foliar application of potassium silicate induces metabolic changes in strawberry plants, Journal of Plant Nutrition, 21:(1) 157-167.

William, H.B. and M. Jerry, 2017. Carboxylic acid, Chemical component. Encyclopedia Britanica. https://ww.britanica. Com/science/carboxylic. acid.

Williams, D.G., R.N. Mack, and R.A. Black, 1995. Ecophysiology of introduced Pennisetum setaceum on Hawaii: the role of phenotypic plasticity. Ecology, 76:1569-1580. 